Review began 11/16/2021 Review ended 12/04/2021 Published 12/07/202

๑) Copyright 2021 Altuwaijri et al. This is an open access article distributed under the terms of the Creative Commons Attribution License BY 4.0 , which permits unrestricted use BY. 4., which permits unrestricted distribution, and reproduction in any

\section{Prevalence of Metastasis and Involvement of Level IV and V in Oral Squamous Cell Carcinoma: A Systematic Review}

Ahmad A. Altuwaijri ${ }^{1,2}$, Turki M. Aldrees ${ }^{3}$, Mohammed A. Alessa ${ }^{1}$

1. Department of Otolaryngology-Head and Neck Surgery, College of Medicine, King Saud University, Riyadh, SAU 2. Department of Otolaryngology-Head and Neck Surgery, Security Forces Hospital, Riyadh, SAU 3. Department of Otolaryngology-Head and Neck Surgery, College of Medicine, Prince Sattam Bin Abdulaziz University, Alkhari, SAU

Corresponding author: Ahmad A. Altuwaijri, aamtu2@gmail.com

\section{Abstract}

The occurrence of occult metastases in oral cavity squamous cell carcinoma (OSCC) to lower levels in the neck (levels IV and V) or development of skip metastases that bypass the upper neck levels (levels I to III) and go directly to level IV or V is common. This challenges the efficacy of conventional neck dissection approaches in the treatment of OSCC. Therefore, the decision to include lower levels cervical nodes during elective neck dissection of OSCC remains controversial.

This systematic review was designed to assess the prevalence of level IV and/or V involvement or skip metastases in patients with the clinically negative neck (cN0) or positive $(\mathrm{cN}+)$ oral squamous cell carcinoma (OSCC). We searched for studies published between December 2000 and December 2020. Potentially relevant abstracts and full-text articles were screened, and data from the studies were extracted. Quality was rated using the Newcastle Ottawa Scale (NOS) criteria.

In total, 802 abstracts and 227 full-text articles were screened, and 32 studies were included in this analysis. The prevalence of metastasis ranged from $1.8 \%$ to $66.0 \%$. The incidence for skip metastasis to level IV or V was low, reaching $8.5 \%$. Evidence favored elective neck dissection, including levels I to III, in selected patients with OSCC and patients with $\mathrm{cNO}$ or $\mathrm{cN}+$ neck. The literature was non-conclusive on the recommendation for inclusion of lower levels.

Categories: Otolaryngology, Pathology, Oncology

Keywords: supraomohyoid neck dissection, selective neck dissection, skip metastasis, level v, level iv, metastasis, elective neck dissection, oral squamous cell carcinoma

\section{Introduction And Background}

Oral squamous cell carcinoma (OSCC), constituted by a broad range of tumors with diverse etiologies, is a life-threatening malignant tumor that ranks as the sixth most common cancer by incidence, with 500,000 new cases reported worldwide annually, accounting for $32 \%-40 \%$ of all head and neck cancers $[1,2]$. It can metastasize to cervical lymph nodes via lymphatic vessels [2,3], with neck metastasis being the most important prognostic factor which affected survival by a nearly $50 \%$ decline [4]. The incidence of clinica cervical metastases from OSCC has been found to occur in as many as $40 \%$ of cases [5]. Moreover, occul regional lymph node metastases incidence detected using histopathological and immunohistochemical methods was found to range between $15 \%$ and 34\% [6] among patients without clinical or radiologic evidence of lymph node metastases preoperatively.

Selective neck dissection (SND), which removes lymph node groups at designated anatomic levels (I-III), is accepted as the standard of care for the management of regional disease in OSCC patients with clinically positive node $\left(\mathrm{cN}^{+}\right)$involvement $[7,8]$, as well as the standard elective procedure for clinically node-negative (cN0) patients or those with microscopic disease [9,10], resulting in improved quality of life and a lower likelihood of orofacial complication or shoulder dysfunction compared to other modalities, including comprehensive neck dissection, such as modified radical neck dissection (MRND) or radical neck dissection (RND) $[11,12]$. However, several studies have concluded that supraomohyoid neck dissection (SOHND, level I-III) is inadequate in patients with OSCC, owing to occult metastasis to neck level IV and that this leve should be routinely dissected $[13,14]$.

In view of the controversies surrounding the inclusion of lower levels for dissection, the present study wa designed with the objectives of conducting a systematic review of all relevant published literature: (i) to study the prevalence and distribution of metastasis levels and related adverse outcomes in clinically N0 and $\mathrm{N}+$ OSCC; and (ii) to determine the frequency of involvement of levels IV and V, as well as skip metastasis to level IV in patients diagnosed with OCSCC without preoperative evidence of neck involvement. We aimed to summarize the recommendations for routine dissection of lower levels of nodes in patients with OSCC.

\section{Review}

\section{Methodology}

Search Strategy

A comprehensive search for all relevant articles published in English between January 2000 and Decembe 2020 was performed using the electronic databases PubMed, Embase, Ovid, Google Scholar, and Science Direct. We included retrospective, prospective, clinical trials, and cross-sectional studies. The key search terms used either alone or in combination were neck dissection, radical neck dissection, cN0 neck, cN+ neck, oral squamous cell carcinoma, skip metastasis, occult metastasis, lymph node management, neck metastasis, oral cavity cancer, and tongue cancer. The references of articles and citations were also searched 


\section{Cureus}

for additional potentially relevant publications.

Study Eligibility Criteria

All studies that included patients who underwent a neck dissection (ND) of at least levels I through III or I-IV and presented information on clinically node-negative ( $\mathrm{cNO}$ ) and/or clinically node-positive $\left(\mathrm{cN}^{+}\right)$necks

were eligible for inclusion. The inclusion criteria were as follows: (1) any prospective or retrospective cohort, (2) a study population with the histopathologic diagnosis of OSCC, and (3) full text available in the English language. In addition, studies that reported skip metastasis (metastasis solely at neck level IV or V) were also

eligible for inclusion. Exclusion criteria were as follows: (1) studies on patients who underwent treatment other than surgery as primary treatment, such as preoperative radiotherapy and chemotherapy, and (2) studies on recurrent tumors or tumors other than SCC.

Data Extraction

Information regarding patient characteristics, primary tumor site, treatment, sample size, metastasis, authors, publication year, and the country was retrieved from the selected articles. Data were initially extracted and evaluated by two authors (AA, TA). The distributions of the T category, the extent of ND, the subsite of the primary tumor, and nodal metastasis were recorded. A skip metastasis was defined as a positive level IV (or lower) node on final pathology without the involvement of higher levels (i.e., levels IIII). A level IV nodal metastasis coexisting with nodes at other neck levels was assessed separately. We followed the Preferred Reporting Items for Systematic Reviews and Meta-Analyses (PRISMA) guidelines for reporting the included observational studies [15].

Quality Evaluation

The quality of literature was evaluated according to the Newcastle Ottawa Scale (NOS) evaluation criteria [16]. By quality evaluation, 21 references were ranked high, seven references were medium, and only four were ranked low (Table 1 ). 


\section{Cureus}

\begin{tabular}{|c|c|c|}
\hline Author & Year & NOS quality rating \\
\hline Silverman [17] & 2003 & 8 \\
\hline Anderson [18] & 2002 & 7 \\
\hline Jena [19] & 2013 & 7 \\
\hline Liao [20] & 2011 & 6 \\
\hline Jayasuriya [21] & 2020 & 8 \\
\hline Haranadha [22] & 2018 & 7 \\
\hline Chheda [23] & 2014 & 7 \\
\hline Kakei [24] & 2020 & 8 \\
\hline Marchiano [25] & 2016 & 4 \\
\hline Givi [26] & 2012 & 5 \\
\hline Pandey [27] & 2018 & 7 \\
\hline Agarwal [28] & 2018 & 3 \\
\hline Mishra [29] & 2010 & 6 \\
\hline Shimura [30] & 2019 & 7 \\
\hline Parikh [31] & 2013 & 6 \\
\hline Jerjes [32] & 2010 & 6 \\
\hline Cariati [33] & 2018 & 7 \\
\hline Patel [34] & 2019 & 5 \\
\hline Lodder [35] & 2008 & 5 \\
\hline Lim [36] & 2006 & 6 \\
\hline Kowalski [37] & 2002 & 7 \\
\hline Feng [38] & 2013 & 8 \\
\hline Sivanandan [39] & 2004 & 7 \\
\hline Crean [40] & 2003 & 4 \\
\hline Khafif [41] & 2001 & 6 \\
\hline Balasubramanian [42] & 2012 & 7 \\
\hline Köhler [43] & 2018 & 8 \\
\hline Deo [44] & 2007 & 7 \\
\hline de Vicente [45] & 2015 & 7 \\
\hline Rani [46] & 2015 & 3 \\
\hline Chatterjee [47] & 2019 & 6 \\
\hline Vishak [48] & 2014 & 7 \\
\hline
\end{tabular}

TABLE 1: The quality rating of included studies using the Newcastle Ottawa Scale (NOS)

\section{Results}

The search and selection process of the articles is presented in Figure 1. A total of 1482 articles were identified via the database search based on the selection criteria, and two additional articles were later found through reviewing articles and reference lists of retrieved articles. After removing duplicates, 453 articles were screened by their titles and abstracts, and 61 were retained. After full-text revision, 31 articles were excluded (Figure 1). Thus, 32 studies [17-48], all published in English, were included for further analysis. 


\section{Cureus}

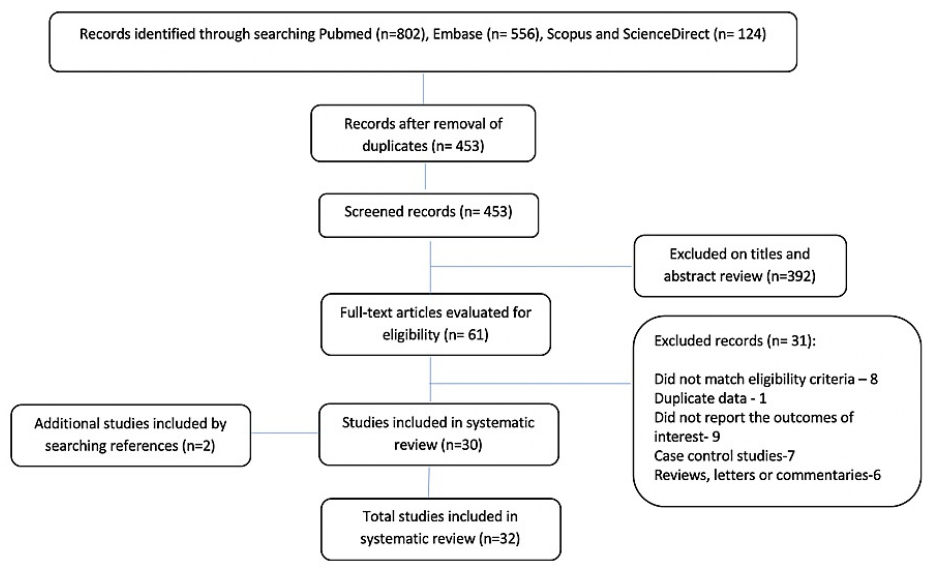

FIGURE 1: PRISMA flowchart: selection of studies for systematic review PRISMA, Preferred Reporting Items for Systematic Reviews and Meta-Analyses

Description of the Studies

Data of 12,309 patients included in the 32 studies were analyzed. In all studies, cases of level IV or V metastasis and cervical IIb metastasis were confirmed by pathologic examination or other technologies. All studies did not, however, have consistent inclusion criteria and exclusion criteria. Five studies

$[19,23,28,30,40]$ reported data from only OSCC patients with cN0, while three $[18,21,24]$ had only data on cN+; five studies $[17,29,31,33,35]$ had mixed data of clinical N0 and N+ cases. The details of the studies included are summarized in Table 2 .

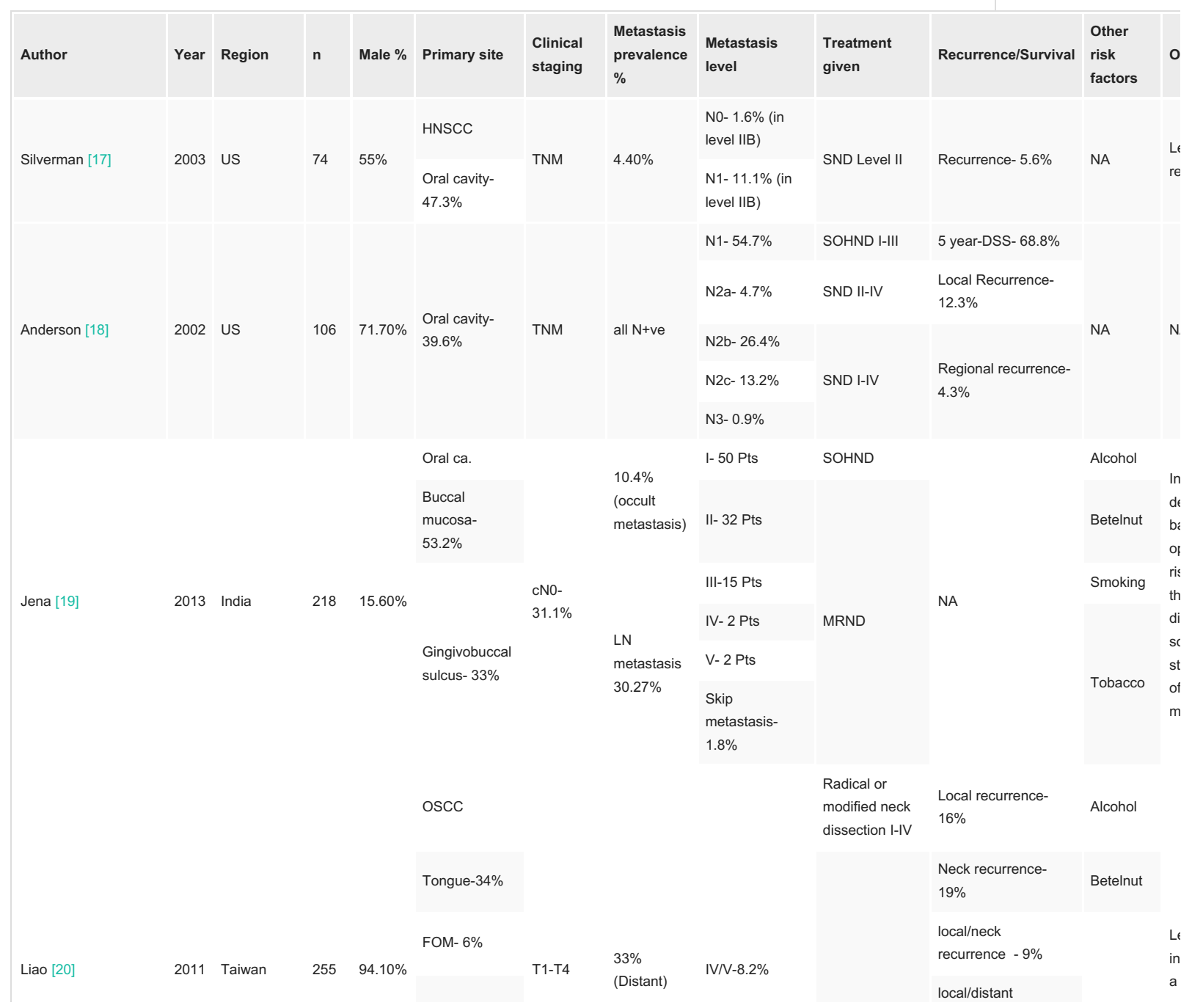




\section{Cureus}

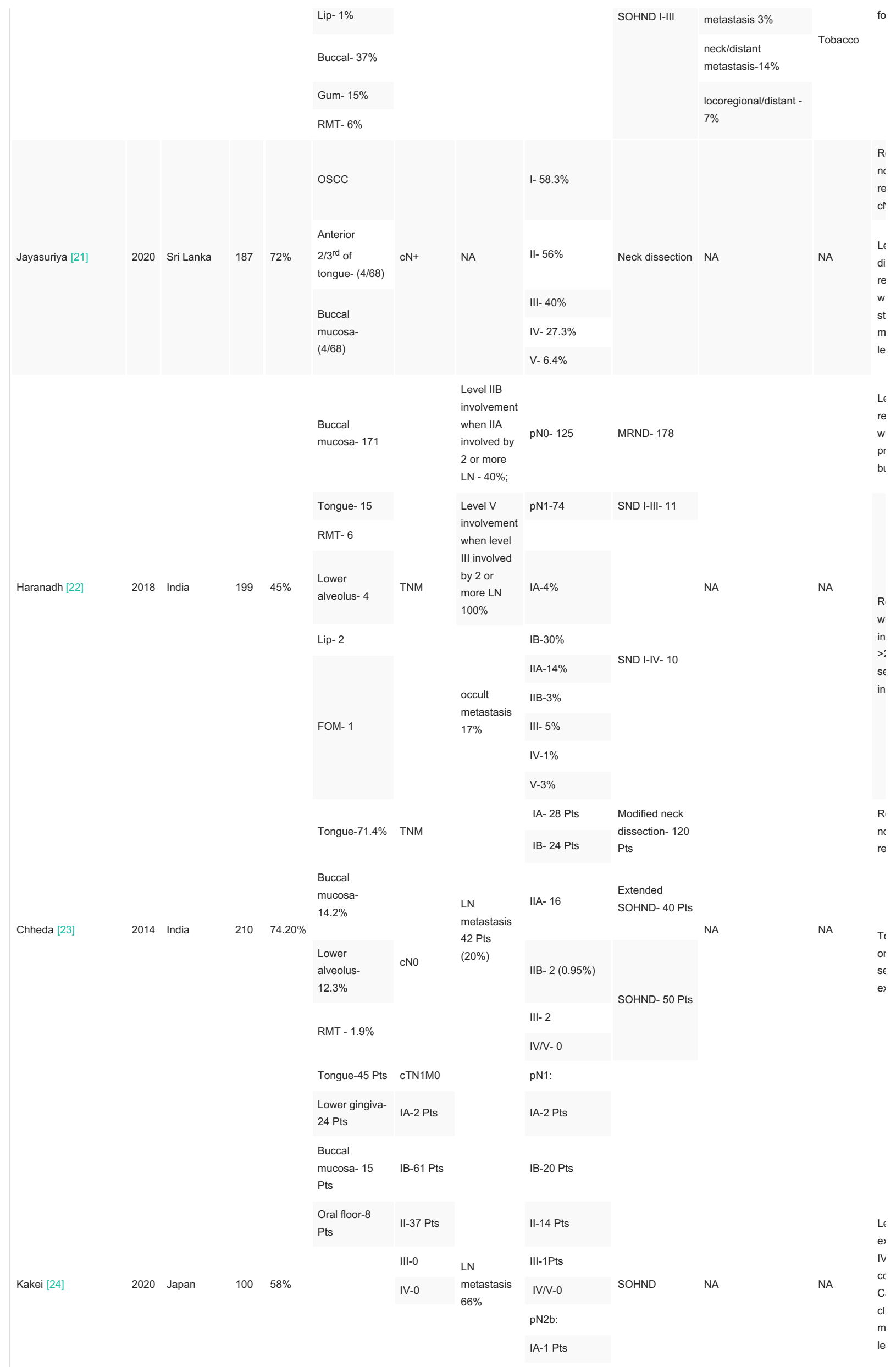




\section{Cureus}

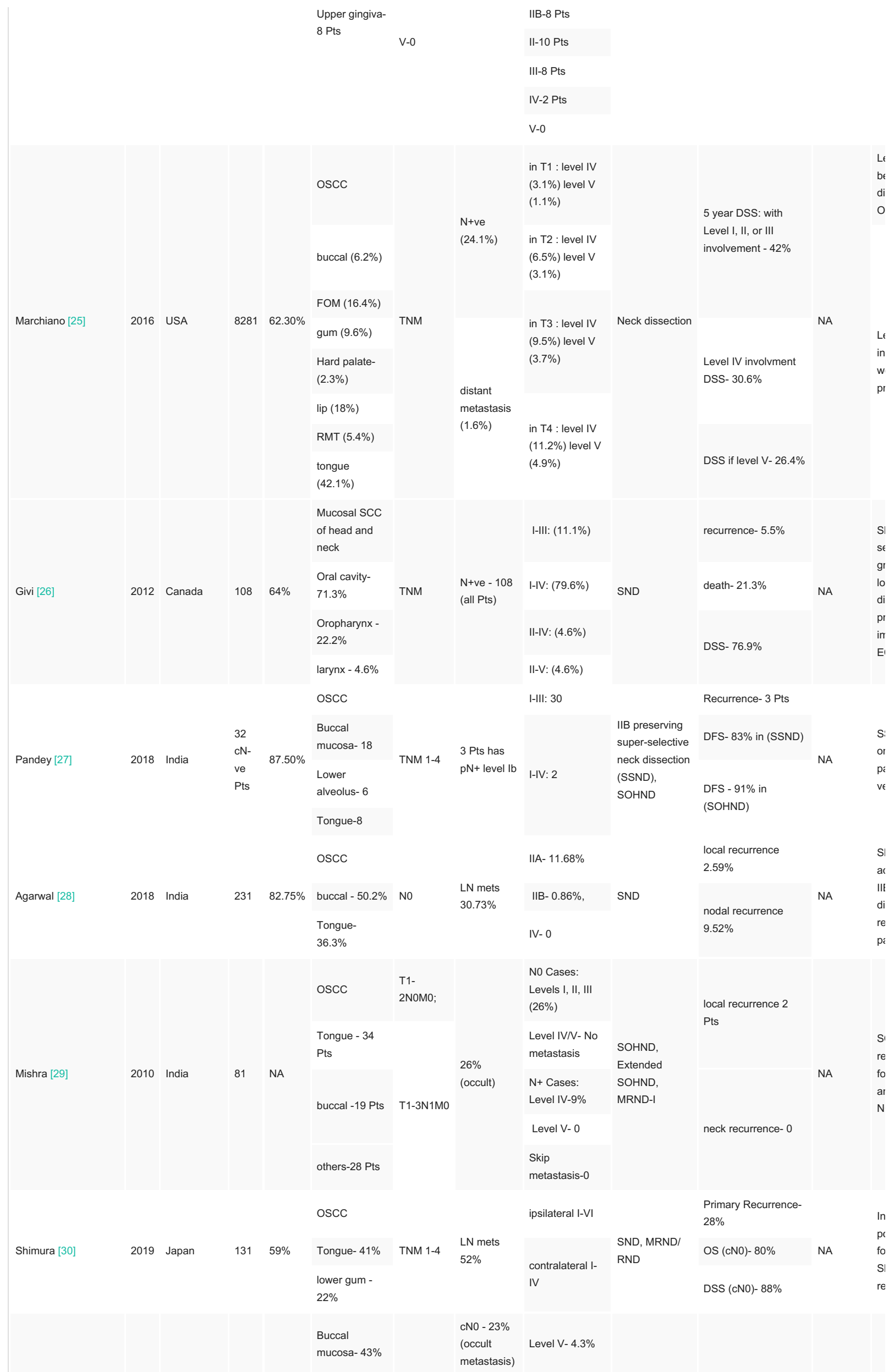




\section{Cureus}

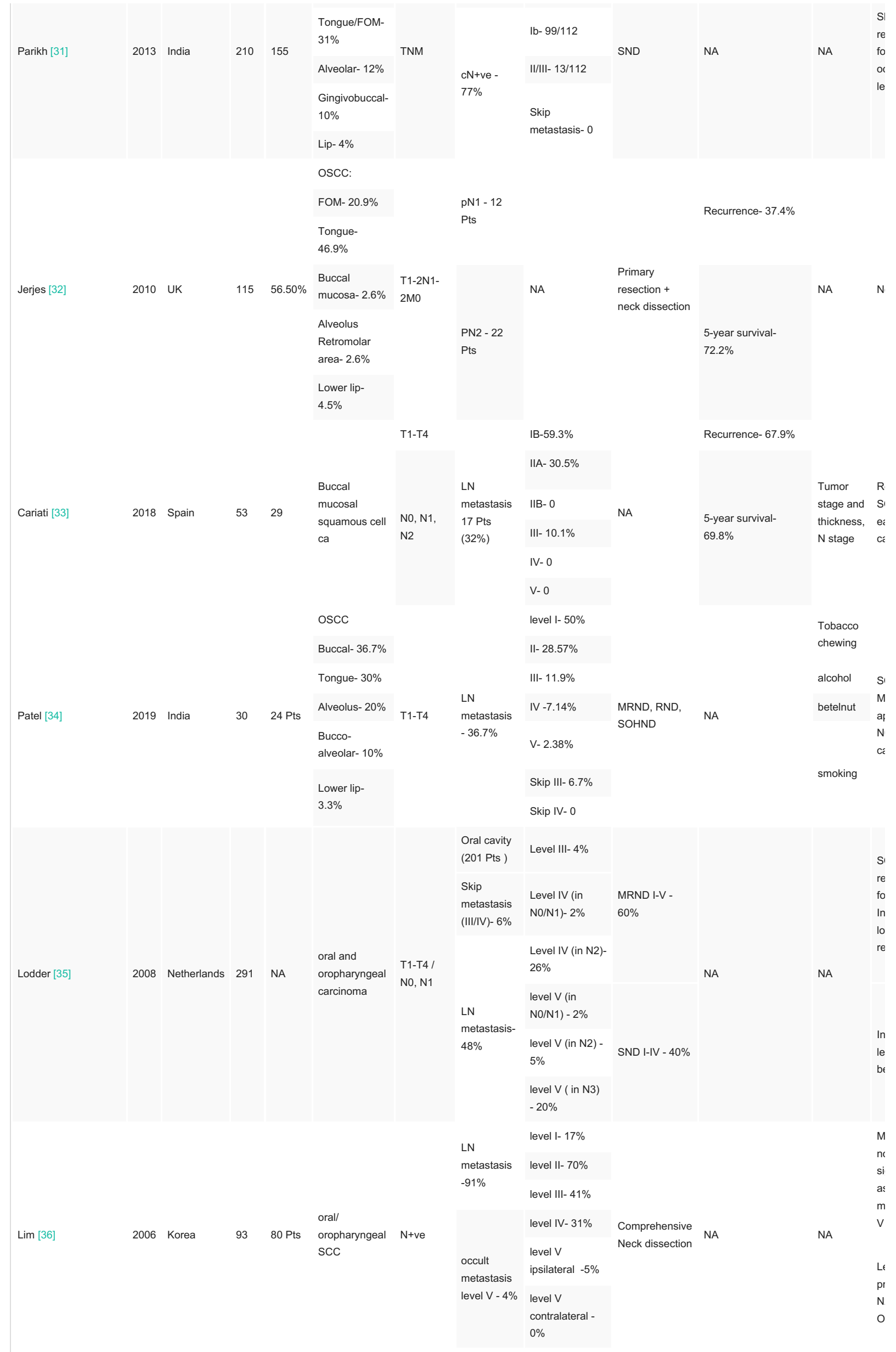




\section{Cureus}

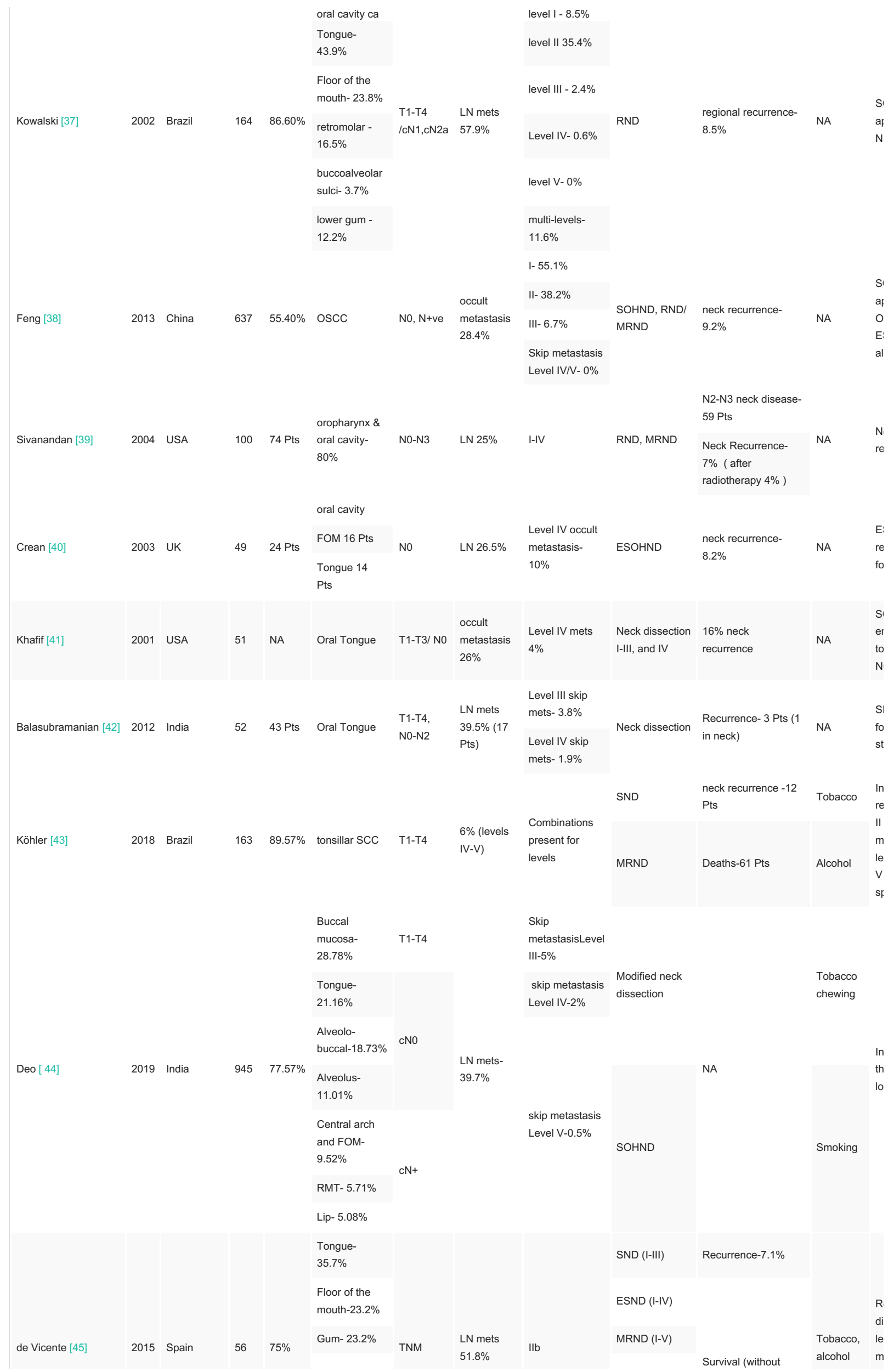




\section{Cureus}

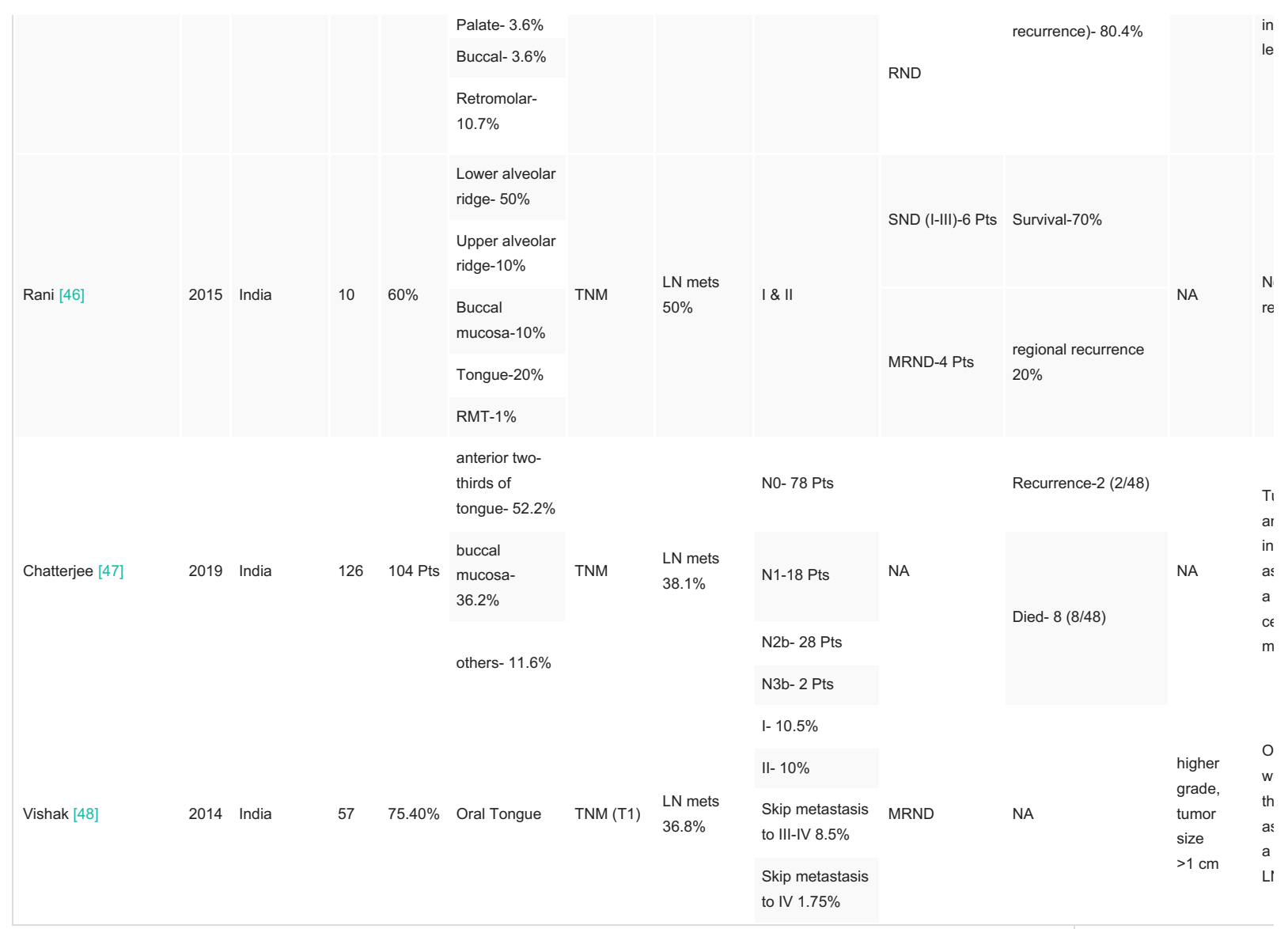

TABLE 2: Study characteristics and pattern of lymph node metastasis in oral cavity squamous cell carcinoma

SCC, squamous cell carcinoma; HNSCC, head and neck SCC; OSCC, oral cavity SCC; TNM, tumor-node-metastasis staging system; SND, selective neck dissection; SOHND, supraomohyoid neck dissection; SSND, super-selective neck dissection; ESOND, extended supraomohyoid neck dissection; MRND, modified radical neck dissection; RND, radical neck dissection; cN/pN, clinical lymph node status/pathological lymph node status; FOM, floor of mouth; RMT, retromolar trigone; DSS, disease-specific survival; LN, lymph nodes; Ca, cancer; mets, metastasis; Pts, patients.

The prevalence of metastasis ranged from $1.8 \%$ to $66.0 \%$ [24]. Among 23 studies reporting metastasis level up to level V, 13 studies [19-22,24,29,34,35,37,40-43] reported level IV involvement, and eight reported level V involvement [19-22,31,34,36,43]. The rate of involvement of level IV among the patients with cN0 was up to $10.4 \%$ [19], with four studies [23,28,29,33] reporting no involvement.

Six articles $[19,29,31,34,38,48]$ illustrated the characteristics of cervical skip metastasis patients, which gave details of sites, T stages, isolated IIb metastases [45], and associated metastatic lymph nodes. The incidence for skip metastasis to level IV or V was low, reaching up to $8.5 \%[29,31,34,48]$. However, not all the information was complete for each study. The most common primary site for level IIb metastases was the tongue $[22-24,45,47]$, reported between $2 \%$ and $28 \%$ [23,47]. The rate of skip metastasis among cN0 was also low, reaching $1.8 \%[19,29,31]$.

Studies Recommending Dissection of Lower Levels

Five studies $[17,21,24,45,48]$ recommended dissection of lower neck levels. Three of these studies $[21,24,48]$ reported metastasis to level IV, while one [17] reported metastasis to level V. None of them were on patients with cN0, two $[21,24]$ had data on $\mathrm{N}^{+}$, while three $[17,45,48]$ had mixed data. One study reported metastasis to level IIb in tongue carcinoma [45].

Studies Not Recommending Dissection of Lower Levels

Thirteen studies [21,22,24,28-31,35-37] did not recommend dissection of lower neck levels because of the low prevalence of metastasis to these levels. Only six of these studies [28-31,35,37] reported metastasis to level IV, while five studies $[21,22,24,35,36]$ reported metastasis to level V. Four of them were on patients with cN0 $[23,28,29,31]$, while six $[21,24,29-31,36]$ presented data on $\mathrm{N}+$ patients. Three studies $[22,35,37]$ reported mixed nodal status, and one study [23] was on level IIb involvement for oral tongue carcinoma.

Studies With Inconclusive Results on Dissection of Lower Levels

Few studies $[18,19,34,39,41,47]$ were inconclusive in recommending whether lower-level dissections should be undertaken or not, with routine neck dissections. These studies reported no metastasis at level IV or V 
and concluded that SND I-III was sufficient in most cases. However, these studies also went on to recommend dissection of levels IV and V based on the surgeons' clinical decisions during surgery. Of these, one [19] reported data on cN0 neck, one [18] on $\mathrm{N}+$ neck, and four $[34,39,41,47]$ had mixed nodal status. In addition, twelve studies $[20,25-27,32,33,38,40,42-44,46]$ did not make any clear recommendation on inclusion or non-inclusion of lower levels for neck dissections for lack of such data. A study by Jayasuriya et al. [21] presented ambiguous results wherein the authors did not recommend routine neck dissection for level V; however, they went on to recommend level V dissection when nodal stages $>\mathrm{N} 2 \mathrm{~b}$ and metastasis to level II and IV were observed in a case.

\section{Discussion}

This review revealed that the available literature favored either selective neck dissection, including only the upper levels (I-III), or was inconclusive. Most studies support the view that primary neck dissections should be limited to upper levels only, owing to the low rates of lower level (level IV and beyond) metastasis and the difficulty as well as the damage incurred (thereby introducing complications) due to the inclusion of those levels. Through independent studies, most authors have supported that high efficacy and minor morbidity for selecting pN+ OSCC patients may be achievable using SND (I-III) $[38,49,50]$. In a meta-analysis that compared SND with MRND/RND in OSCC patients with $\mathrm{CN}+$ disease, authors [51] suggested that $\mathrm{CN}+\mathrm{OSCC}$ patients treated with SND (I, I-III, or I-IV) or those treated with MRND/RND had comparable clinical outcomes measured by no significant difference for regional recurrence, overall survival (OS), or diseasespecific survival (DSS) between any of the dissection treatment types. The meta-analysis was, however, limited by the inclusion of studies where the extent and selection of the SND levels differed between studies other than levels I-II. The result of this meta-analysis supports our claim that even with variable surgical methods, it is not advisable to routinely include lower-level dissections. Contrary to the findings of the present study, independent studies, such as one by Shah et al. [52], have reported that $15 \%-16 \%$ of tongue/oral cancer with clinically detected lymph node(s) (cLN(s)) had pathological lymph node(s) (pLN(s)) to level IV, thereby recommending extended SOHND, which includes dissecting level IV.

Skip metastasis, described by Byers et al. [14], refers to the condition in which OSCC bypasses levels I, II, or both and goes directly to levels III or IV. The rate of skip metastasis in the original study was reported as $15.8 \%$, thereby recommending routine dissection at neck level IV. Later analysis, however, revealed that among cN0 patients, only 5.5\% had skip metastasis to level IV, making the recommendations controversial. Later, Crean et al. [40] similarly demonstrated that $10 \%$ of patients had involvement of neck level IV despite having been preoperatively diagnosed with a cNO neck, with only $2 \%$ having a true skip metastasis to level IV. In a recent meta-analysis, the authors found the rate of skip metastasis to be low (overall involvement rate of $2.53 \%$ and skip metastasis rate of $0.50 \%$ ), even with advanced tumor stages, wherein the final recommendation was not to include dissection of lower levels routinely [53]. A meta-analysis was conducted in 2020 to investigate the prevalence of level IV involvement and skip metastases in patients with clinically negative neck (cN0) oral tongue squamous cell carcinoma. It also recommended elective neck dissection that includes levels I to III because of the low rates of level IV involvement and skip metastasis [54]. Our review also supports the view for non-inclusion of lower levels in ND for suspicion of skip metastasis.

Some arguments may be made in terms of benefits archived in ipsilateral, contralateral, or bilateral node infiltration. Although we did not study the laterality of recurrence, the available literature [30] suggested that SND (I-III) could achieve good regional control and had a favorable prognosis for cN+ OSCC. In a study with ipsilateral neck recurrence rates ranging from $11 \%-14 \%$, similar conclusions were drawn for the $\mathrm{pN}+$ cohort [30].

Some studies reported data on oral tongue SCC, which is the most common primary site for OSCC, with most studies suggesting metastasis to level IIb $[55,56]$, leading scholars to recommend level IIB dissection routinely in tongue SCC. Few studies $[57,58]$ found no statistical significance between site and metastasis, which makes a contrary view due to the difficulty of approach, questionable benefits, and avoidance of postoperative shoulder disability [8]. Even with regards to level IV metastasis, most studies present a reserved view to include lower-level dissection as an exception for tongue carcinoma [14]. Our study found that all included literature for oral tongue carcinoma recommended lower-level dissection, probably owing to the tendency of tongue cancer toward early metastasis, the possible reason being that the tongue possesses an extensive lymphatic network.

\section{Strengths and limitations}

The present review included studies that reported varied study groups and regions, thereby introducing heterogeneity. The heterogeneity of study groups is considered an important confounder. In our case, it resulted in the lack of appropriate data stratification by $\mathrm{T}$ stage, subsites, and involvement of other neck levels that we could not address. The retrospective nature of the included studies also introduced bias, which could not be addressed. However, we exercised caution in including studies with primary neck dissection data only. We excluded all studies with patients with revision NDs and omitted all groups lacking this information to eliminate bias from combining the results of the primary neck surgery with those of revision surgeries for neck recurrences, which may falsely inflate the rate of level IV or lower-level involvement. While most studies presented mixed data for $\mathrm{cN} 0$ and $\mathrm{cN}+$ necks, we segregated data wherever possible to report the differences according to nodal status. Lastly, the decision for SND or MRND techniques is widely debated due to the lack of universally accepted guidelines for the anatomic limits for the variety of SND procedures available. The exact anatomic boundaries for an SND are also thought to vary among institutions and even among surgeons within an institution [59]. The analysis of these differences could not be accounted for in the present review.

\section{Conclusions}

OSCC is constituted by a broad range of tumors with diverse etiologies. It can metastasize to cervical lymph nodes via lymphatic vessels. SND is considered a standard of care for most subsites, even in early-stage disease. Based on the evidence reviewed in the present study, the frequency of lower-level metastasis (level IV or V), as well as skip metastasis in OSCC, was low. Hence, routine dissection of these levels in cN0 and $\mathrm{cN}+$ necks may be avoided except for tongue cancer. Since dissection of level IV/V is a burden with extra 
time and might expose patients to more complications, dissection might be selected for specific subsites and extension. It is recommended to dissect level IIb and lower levels for tongue cancers without considering the stage of primary lesions or lymph node status. Most studies recommended sparing lower-level neck dissections, while some were inconclusive.

\section{Additional Information \\ Disclosures}

Conflicts of interest: In compliance with the ICMJE uniform disclosure form, all authors declare the following: Payment/services info: All authors have declared that no financial support was received from any organization for the submitted work. Financial relationships: All authors have declared that they have no financial relationships at present or within the previous three years with any organizations that might have an interest in the submitted work. Other relationships: All authors have declared that there are no other relationships or activities that could appear to have influenced the submitted work.

\section{References}

1. Kamangar F, Dores GM, Anderson WF: Patterns of cancer incidence, mortality, and prevalence across five continents: defining priorities to reduce cancer disparities in different geographic regions of the world. J Clin Oncol. 2006, 24:2137-50. 10.1200//CO.2005.05.2308

2. Massano J, Regateiro FS, Januário G, Ferreira A: Oral squamous cell carcinoma: review of prognostic and predictive factors. Oral Surg Oral Med Oral Pathol Oral Radiol Endod. 2006, 102:67-76. 10.1016/i.tripleo.2005.07.038

3. Kumar T, Patel MD: Pattern of lymphatic metastasis in relation to the depth of tumor in oral tongue cancers: a clinico pathological correlation. Indian J Otolaryngol Head Neck Surg. 2013, 65:59-63. 10.1007/s12070-012-0504-y

4. Amit M, Yen TC, Liao CT, et al.: Clinical nodal stage is a significant predictor of outcome in patients with oral cavity squamous cell carcinoma and pathologically negative neck metastases: results of the international consortium for outcome research. Ann Surg Oncol. 2013, 20:3575-81. 10.1245/s10434-0133044-0

5. Okura M, Aikawa T, Sawai NY, Iida S, Kogo M: Decision analysis and treatment threshold in a management for the N0 neck of the oral cavity carcinoma. Oral Oncol. 2009, 45:908-11. 10.1016/j.oraloncology.2009.03.013

6. Noguti J, De Moura CF, De Jesus GP, Da Silva VH, Hossaka TA, Oshima CT, Ribeiro DA: Metastasis from oral cancer: an overview. Cancer Genomics Proteomics. 2012, 9:329-35.

7. Ferlito A, Rinaldo A: Is radical neck dissection a current option for neck disease? . Laryngoscope. 2008, 118:1717-8. 10.1097/MLG.0b013e31817d9cd5

8. Pantvaidya GH, Pal P, Vaidya AD, Pai PS, D'Cruz AK: Prospective study of 583 neck dissections in oral cancers: implications for clinical practice. Head Neck. 2014, 36:1503-7. 10.1002/hed.23494

9. Manni JJ, van den Hoogen FJ: Supraomohyoid neck dissection with frozen section biopsy as a staging procedure in the clinically node-negative neck in carcinoma of the oral cavity. Am J Surg. 1991, 162:373-6. 10.1016/0002-9610(91)90151-3

10. Brazilian Head and Neck Cancer Study Group: Results of a prospective trial on elective modified radical classical versus supraomohyoid neck dissection in the management of oral squamous carcinoma. Am J Surg. 1998, 176:422-7. 10.1016/s0002-9610(98)00230-x

11. Spalthoff S, Zimmerer R, Jehn P, Gellrich NC, Handschel J, Krüskemper G: Neck dissection's burden on the patient: functional and psychosocial aspects in 1,652 patients with oral squamous cell carcinomas. J Oral Maxillofac Surg. 2017, 75:839-49. 10.1016/j.joms.2016.09.037

12. Chepeha DB, Taylor RJ, Chepeha JC, et al.: Functional assessment using Constant's Shoulder Scale after modified radical and selective neck dissection. Head Neck. 2002, 24:432-6. 10.1002/hed.10067

13. De Zinis LO, Bolzoni A, Piazza C, Nicolai P: Prevalence and localization of nodal metastases in squamous cell carcinoma of the oral cavity: role and extension of neck dissection. Eur Arch Otorhinolaryngol. 2006, 263:1131-5. 10.1007/s00405-006-0128-5

14. Byers RM, Weber RS, Andrews T, McGill D, Kare R, Wolf P: Frequency and therapeutic implications of "skip metastases" in the neck from squamous carcinoma of the oral tongue. Head Neck. 1997, 19:14-9. 10.1002/(sici) 1097-0347(199701)19:1<14::aid-hed3>3.0.co;2-y

15. Moher D, Liberati A, Tetzlaff J, Altman DG: Preferred reporting items for systematic reviews and metaanalyses: the PRISMA statement. PLoS Med. 2009, 6:e1000097. 10.1371/journal.pmed.1000097

16. The Newcastle-Ottawa Scale (NOS) for assessing the quality of nonrandomised studies in meta-analyses (2021). http://www.ohri.ca/programs/clinical_epidemiology/oxford.asp.

17. Silverman DA, El-Haji M, Strome S, Esclamado RM: Prevalence of nodal metastases in the submuscular recess (level IIb) during selective neck dissection. Arch Otolaryngol Head Neck Surg. 2003, 129:724-8. 10.1001/archotol.129.7.724

18. Andersen PE, Warren F, Spiro J, et al.: Results of selective neck dissection in management of the nodepositive neck. Arch Otolaryngol Head Neck Surg. 2002, 128:1180-4. 10.1001/archotol.128.10.1180

19. Jena A, Patnayak R, Vamsi RN, Reddy SK, Banoth M: Surgical management of the neck in oral cancers: a single institute experience from South India. Arch Int Surg. 2013, 3:106-11. 10.4103/2278-9596.122928

20. Liao CT, Lee LY, Huang SF, et al.: Outcome analysis of patients with oral cavity cancer and extracapsular spread in neck lymph nodes. Int J Radiat Oncol Biol Phys. 2011, 81:930-7. 10.1016/j.ijrobp.2010.07.1988

21. Jayasuriya NS, Mannapperuma NT, Siriwardana S, et al.: Incidence of metastasis to level V lymph nodes in clinically positive necks among Sri Lankan patients with oral squamous cell carcinoma. Br J Oral Maxillofac Surg. 2021, 59:771-5. 10.1016/j.bjoms.2020.09.027

22. Haranadh S, Nandyala R, Bodagala V, Hulikal N: A prospective analysis of prevalence of metastasis in levels IIB and V neck nodes in patients with operable oral squamous cell carcinoma. Oral Oncol. 2018, 83:115-9. 10.1016/i.oraloncology.2018.06.020

23. Chheda YP, Pillai SK, Parikh DG, Dipayan N, Shah SV, Alaknanda G: A prospective study of level IIB nodal metastasis (supraretrospinal) in clinically NO oral squamous cell carcinoma in Indian population. Indian J Surg Oncol. 2017, 8:105-8. 10.1007/s13193-014-0359-7

24. Kakei Y, Komatsu H, Minamikawa T, et al.: Extent of neck dissection for patients with clinical N1 oral cancer. Int J Clin Oncol. 2020, 25:1067-71. 10.1007/s10147-020-01635-8

25. Marchiano E, Patel TD, Eloy JA, Baredes S, Park RC: Impact of nodal level distribution on survival in oral cavity squamous cell carcinoma: a population-based study. Otolaryngol Head Neck Surg. 2016, 155:99-105. $10.1177 / 0194599816636356$

26. Givi B, Linkov G, Ganly I, et al.: Selective neck dissection in node-positive squamous cell carcinoma of the head and neck. Otolaryngol Head Neck Surg. 2012, 147:707-15. 10.1177/0194599812444852

27. Pandey M, Karthikeyan S, Joshi D, Kumar M, Shukla M: Results of a randomized controlled trial of level IIb 
preserving neck dissection in clinically node-negative squamous carcinoma of the oral cavity. World J Surg Oncol. 2018, 16:219. 10.1186/s12957-018-1518-z

28. Agarwal SK, Akali NR, Sarin D: Prospective analysis of 231 elective neck dissections in oral squamous cell carcinoma with node negative neck-to decide the extent of neck dissection. Auris Nasus Larynx. 2018, 45:156-61. 10.1016/j.anl.2017.05.019

29. Mishra P, Sharma AK: A 3-year study of supraomohyoid neck dissection and modified radical neck dissection type I in oral cancer: with special reference to involvement of level IV node metastasis. Eur Arch Otorhinolaryngol. 2010, 267:933-8. 10.1007/s00405-009-1155-9

30. Shimura S, Ogi K, Miyazaki A, et al.: Selective neck dissection and survival in pathologically node-positive oral squamous cell carcinoma. Cancers (Basel). 2019, 11:269. 10.3390/cancers11020269

31. Parikh DG, Chheda YP, Shah SV, Patel AM, Sharma MR: Significance of level v lymph node dissection in clinically node positive oral cavity squamous cell carcinoma and evaluation of potential risk factors for level v lymph node metastasis. Indian J Surg Oncol. 2013, 4:275-9. 10.1007/s13193-013-0241-z

32. Jerjes $\mathrm{W}$, Upile T, Petrie A, et al.: Clinicopathological parameters, recurrence, locoregional and distant metastasis in 115 T1-T2 oral squamous cell carcinoma patients. Head Neck Oncol. 2010, 2:9. 10.1186/17583284-2-9

33. Cariati P, Cabello Serrano A, Marin Fernandez A, Julia Martinez MA, Fernandez Solis J, Martinez Lara I: Behavior of buccal mucosal squamous cell carcinoma: a retrospective study of 53 carcinomas of this anatomical region. Craniomaxillofac Trauma Reconstr. 2019, 12:8-13. 10.1055/s-0038-1668583

34. Patel S, Singh I, Gulati A, Khurana N: A study on neck nodes in oral cancers, with special reference to skip metastasis. Indian J Otolaryngol Head Neck Surg. 2019, 71:474-81. 10.1007/s12070-018-1360-1

35. Lodder WL, Sewnaik A, den Bakker MA, Meeuwis CA, Kerrebijn JD: Selective neck dissection for N0 and N1 oral cavity and oropharyngeal cancer: are skip metastases a real danger?. Clin Otolaryngol. 2008, 33:450-7. 10.1111/j.1749-4486.2008.01781.x

36. Lim YC, Koo BS, Lee JS, Choi EC: Level V lymph node dissection in oral and oropharyngeal carcinoma patients with clinically node-positive neck: is it absolutely necessary?. Laryngoscope. 2006, 116:1232-5 10.1097/01.mlg.0000224363.04459.8b

37. Kowalski LP, Carvalho AL: Feasibility of supraomohyoid neck dissection in N1 and N2a oral cancer patients . Head Neck. 2002, 24:921-4. 10.1002/hed.10127

38. Feng Z, Li JN, Niu LX, Guo CB: Supraomohyoid neck dissection in the management of oral squamous cell carcinoma: special consideration for skip metastases at level IV or V. J Oral Maxillofac Surg. 2014, 72:120311. 10.1016/i.joms.2013.12.008

39. Sivanandan R, Kaplan MJ, Lee KJ, et al.: Long-term results of 100 consecutive comprehensive neck dissections: implications for selective neck dissections. Arch Otolaryngol Head Neck Surg. 2004, 130:136973. 10.1001/archotol.130.12.1369

40. Crean SJ, Hoffman A, Potts J, Fardy MJ: Reduction of occult metastatic disease by extension of the supraomohyoid neck dissection to include level IV. Head Neck. 2003, 25:758-62. 10.1002/hed.10282

41. Khafif A, Lopez-Garza JR, Medina JE: Is dissection of level IV necessary in patients with T1-T3 N0 tongue cancer?. Laryngoscope. 2001, 111:1088-90. 10.1097/00005537-200106000-00029

42. Balasubramanian D, Thankappan K, Battoo AJ, Rajapurkar M, Kuriakose MA, Iyer S: Isolated skip noda metastasis is rare in T1 and T2 oral tongue squamous cell carcinoma. Otolaryngol Head Neck Surg. 2012, 147:275-7. 10.1177/0194599812439664

43. Köhler HF, Franzi SA, Soares FA, Torloni H, Kowalski LP: Distribution of metastatic nodes in NO-1 patients with tonsillar squamous cell carcinoma and its implications for selective neck dissection. Turk Arch Otorhinolaryngol. 2018, 56:139-44. 10.5152/tao.2018.3420

44. Deo S, Singh V, Mokkapati PR, Shukla NK, Dwivedi SN, Sharma A, Biswas A: Clinical spectrum, pattern, and level-wise nodal involvement among oral squamous cell carcinoma patients - audit of 945 oral cancer patient data. Indian J Surg Oncol. 2020, 11:86-91. 10.1007/s13193-019-01011-7

45. de Vicente IC, Rodríguez-Santamarta T, Peña I, Villalaín L, Fernández-Valle Á, González-García M: Relevance of level IIb neck dissection in oral squamous cell carcinoma . Med Oral Patol Oral Cir Bucal. 2015, 20:e547-53. 10.4317/medoral.20491

46. Rani P, Bhardwaj Y, Dass PK, Gupta M, Malhotra D, Ghezta NK: Neck dissection for oral squamous cell carcinoma: our experience and a review of the literature. J Korean Assoc Oral Maxillofac Surg. 2015, 41:299305. 10.5125/ikaoms.2015.41.6.299

47. Chatterjee D, Bansal V, Malik V, et al.: Tumor budding and worse pattern of invasion can predict nodal metastasis in oral cancers and associated with poor survival in early-stage tumors. Ear Nose Throat J. 2019, 98:E112-9. 10.1177/0145561319848669

48. S V, Rohan V: Cervical node metastasis in T1 squamous cell carcinoma of oral tongue- pattern and the predictive factors. Indian J Surg Oncol. 2014, 5:104-8. 10.1007/s13193-014-0301-z

49. Shin YS, Koh YW, Kim SH, Choi EC: Selective neck dissection for clinically node-positive oral cavity squamous cell carcinoma. Yonsei Med J. 2013, 54:139-44. 10.3349/ymj.2013.54.1.139

50. Schiff BA, Roberts DB, El-Naggar A, Garden AS, Myers IN: Selective vs modified radical neck dissection and postoperative radiotherapy vs observation in the treatment of squamous cell carcinoma of the oral tongue. Arch Otolaryngol Head Neck Surg. 2005, 131:874-8. 10.1001/archotol.131.10.874

51. Liang L, Zhang T, Kong Q, Liang J, Liao G: A meta-analysis on selective versus comprehensive neck dissection in oral squamous cell carcinoma patients with clinically node-positive neck. Oral Oncol. 2015, 51:1076-81. 10.1016/j.oraloncology.2015.10.005

52. Shah JP, Candela FC, Poddar AK: The patterns of cervical lymph node metastases from squamous carcinoma of the oral cavity. Cancer. 1990, 66:109-13. 10.1002/1097-0142(19900701)66:1<109::aidcncr2820660120>3.0.co;2-a

53. Warshavsky A, Rosen R, Nard-Carmel N, et al.: Assessment of the rate of skip metastasis to neck level IV in patients with clinically node-negative neck oral cavity squamous cell carcinoma: a systematic review and meta-analysis. JAMA Otolaryngol Head Neck Surg. 2019, 145:542-8. 10.1001/jamaoto.2019.0784

54. Weisz Shabtay N, Ronen O: Level IV neck dissection as an elective treatment for oral tongue carcinoma-a systematic review and meta-analysis. Oral Surg Oral Med Oral Pathol Oral Radiol. 2020, 130:363-72. 10.1016/j.0000.2020.04.810

55. Elsheikh MN, Mahfouz ME, Elsheikh E: Level IIb lymph nodes metastasis in elective supraomohyoid neck dissection for oral cavity squamous cell carcinoma: a molecular-based study. Laryngoscope. 2005, 115:163640. 10.1097/01.mlg.0000176540.33486.c3

56. Maher NG, Hoffman GR: Elective neck dissection for primary oral cavity squamous cell carcinoma involving the tongue should include sublevel IIb. J Oral Maxillofac Surg. 2014, 72:2333-43. 10.1016/.joms.2014.05.02

57. Bhattacharya A, Adwani D, Adwani N, Sharma V: Is it worthy? Removal of level IIB nodes during selective neck dissection (I-III) for oral carcinomas. Ann Maxillofac Surg. 2015, 5:20-5. 10.4103/2231-0746.161052

58. Dabholkar JP, Kapre NM: Level IIb neck dissection in oral cavity cancers- when should one address it..? . Indian J Surg Oncol. 2016, 7:303-6. 10.1007/s13193-015-0461-5

59. Clayman GL, Frank DK: Selective neck dissection of anatomically appropriate levels is as efficacious as modified radical neck dissection for elective treatment of the clinically negatice neck in patients with squamous cell carcinoma of the upper respiratory and digestive tracts. Arch Otolaryngol Head Neck Surg. 


\section{Cureus}

1998, 124:348-53. 10.1001/archotol.124.3.348 